\title{
Bloqueo cardiaco fetal completo. Reporte de dos casos y revisión de la literatura
}

\author{
Carlos Siles G. ${ }^{1}$, Alfredo Hernández A. ${ }^{1,2}$, Alexandra Calvo Q. ${ }^{1}$, Angie Vergara . $^{1}$, \\ Gonzalo Urcelay M. ${ }^{4}$, Jorge Carvajal C. PhD. ${ }^{1,3}$ \\ 1 Departamento de Obstetricia y Ginecología; ${ }^{2}$ Laboratorio de Ultrasonografía; ${ }^{3}$ Laboratorio de Medicina Materno-Fetal; \\ 4 Laboratorio de Cardiología Pediátrica. Facultad de Medicina. Pontificia Universidad Católica de Chile.
}

\section{RESUMEN}

Presentamos 2 casos de embarazos controlados en nuestro servicio con el diagnóstico de bloqueo aurículo-ventricular fetal. Este es un tipo de arritmia poco frecuente, relacionado con la presencia de anticuerpos antiribonucleoproteínas (Ro y La). El manejo es expectante en la mayoría de los casos ya que no existe forma de revertir el bloqueo; en caso de evidenciar una descompensación hemodinámica fetal, se pueden administrar corticoides como medida terapéutica con un éxito limitado. No existe contraindicación del parto vaginal y el uso de $\mathrm{pH}$ de cuero cabelludo y oximetría de pulso parecen ser métodos adecuados para la evaluación de la condición fetal intraparto. Recomendamos el enfoque multidisciplinario en esta patología para evitar intervenciones innecesarias, anticipar los riesgos fetales y obtener un mejor pronóstico postnatal.

\section{PALABRAS CLAVE: Bloqueo aurículo ventricular completo fetal, anti-Ro, anti-La}

\section{SUMMARY}

Here we report the perinatal outcome of two patients with fetal complete atrioventricular (AV) block. This is an uncommon disease, related to the presence of autoantibodies against ribonucleoproteins (Ro and La). Management should be expectant in most cases because a treatment to revert the AV block is not available; when fetal hemodynamic problems are detected corticosteroids can be used, but with limited effectiveness. Vaginal delivery is allowed; fetal scalp pH and pulse oximetry are appropriate for intrapartum fetal surveillance. We recommend a multi disciplinary approach to avoid unnecessary interventions, anticipate fetal risk and obtain a better perinatal outcome.

\section{KEY WORDS: Fetal complete atrioventricular block, anti-Ro, anti-La}

\section{INTRODUCCIÓN}

El bloqueo cardiaco aurículo ventricular (AV) fetal es una arritmia cardiaca debida a una anormalidad del sistema de conducción del corazón fetal; es poco frecuente y se caracteriza por bradicardia, la que puede llevar ocasionalmente a descompensación hemodinámica, hidrops y muerte fetal.

Arritmia cardiaca se define como una alteración del ritmo cardiaco fetal que se mantiene fuera del rango aceptado de 120 a 160 latidos por minuto. No se incluyen las desaceleraciones espontáneas 
o relacionadas con la contracción uterina. La incidencia de alteraciones del ritmo cardiaco se estima en $1-2 \%$ de los embarazos, siendo solo el $10 \%$ fatal para el feto (1). El bloqueo completo tiene una incidencia variable de $1 / 2.000$ a 1/20.000 recién nacidos, sin variaciones entre sexo femenino o masculino (2-4). En Chile la incidencia ha sido descrita en un caso por cada 19.000 nacidos vivos (3). En este artículo reportamos dos casos de bloqueo AV fetal manejados en nuestro servicio, y una revisión de la bibliografía respecto del tema.

\section{Caso 1}

Paciente de 26 años, con un hijo sano nacido por parto vaginal de término 6 años atrás. Inicia control a las 7 semanas, efectuándose una ecografía a las 15 semanas según fecha de la última menstruación que evidencia una gestación única viva con biometría concordante con la edad gestacional por amenorrea. A las 21 semanas se efectuó una segunda ecografía informada como normal, y con un crecimiento en percentil 50 . En su control clínico posterior se detectó una frecuencia cardiaca fetal mantenida de aproximadamente 80 latidos por minuto; la ecografía muestra un feto vivo y activo, de 24 semanas de gestación con bradicardia mantenida (88 lat/min), y es derivada a nuestro centro.

Se efectuó ecocardiografía-Doppler que demostró un bloqueo aurículo-ventricular completo, sin malformaciones estructurales evidentes; con una frecuencia cardiaca auricular de $133 \mathrm{lat} / \mathrm{min}$ y ventricular de 57 lat/min. Se informó un crecimiento fetal en percentil 75-90 para 26 semanas, sin evidencia de hidrops fetal. Se mantuvo en control clínico cada dos semanas, con seguimiento ecográfico de crecimiento y bienestar fetal cada tres semanas, manteniéndose siempre en percentil 75 . Se efectuó ecocardiografía-Doppler a las 28 y 33 semanas que mostraban el bloqueo AV, y ausencia de signos de insuficiencia cardiaca. Entre los exámenes solicitados se evidenció anti-Ro positivo, título de 119,1 U/ $\mathrm{ml}$ (valor normal 0-20) y anti-La negativo.

A las 38 semanas de gestación se realiza un último control ecográfico que evidencia líquido amniótico subjetivamente disminuido, aunque sin constituir oligoamnios. Ante la presencia de buenas condiciones cervicales (Bishop 10) se decide inducción de trabajo de parto mediante infusión endovenosa de ocitocina. Se mantiene a la paciente en monitorización continua de latidos cardíacos fetales, con una frecuencia mantenida de 55 lat $/ \mathrm{min}$, sin desaceleraciones. A las 7 horas de inducción, se asiste parto vaginal de recién nacido masculino,
Apgar 9-9, $3300 \mathrm{~g}$ y talla $50 \mathrm{~cm}$.

El electrocardiograma al nacer mostró bloqueo AV completo, con disociación auriculo-ventricular y frecuencia ventricular de 50-58 lat/min. Evolucionó con frecuencia cardiaca mantenida en 50 lat $/ \mathrm{min}$, por lo que se instaló un marcapasos unicameral con cable ventricular epicárdico, con frecuencia cardiaca de $120 \mathrm{lat} / \mathrm{min}$ al tercer día de vida, evolucionando en forma satisfactoria y siendo dado de alta al sexto día de hospitalización en buenas condiciones generales. Sus controles posteriores han demostrado cavidades cardiacas de tamaño normal para la edad, función sistólica ventricular conservada y buen funcionamiento del marcapasos.

\section{Caso 2}

Paciente de 32 años, multípara de 1, con un hijo sano, sin antecedentes mórbidos de importancia. Evoluciona con un embarazo de curso fisiológico hasta las 28 semanas, cuando se pesquisa durante un control prenatal, frecuencia cardiaca fetal de 80 latidos por minutos. El estudio ultrasonográfico reveló un feto creciendo adecuadamente, activo sin evidencias de hidrops fetal. La ecocardiografíaDoppler fetal reveló un corazón de estructura y función normal con un bloqueo auriculo-ventricular de segundo grado, con una frecuencia auricular de 140 a 150 lat/min y ventricular de 70 a 75 lat/ min. Se realizó estudio inmunológico materno que fue negativo para anticuerpos anti-Ro y anti-La. Se controló semanalmente con ultrasonido mostrando un feto activo, sin signos de hidrops. A las 31 semanas la frecuencia cardiaca fetal disminuye a 50 lat/ min sin signos de insuficiencia cardiaca. Se indica betametasona $12 \mathrm{mg}$ im por 2 veces para inducción de madurez pulmonar fetal. Evaluada a la semana, la frecuencia cardiaca fetal disminuyó a 35-40 lat/ min sin signos de hidrops fetal. Se indica la interupción por cesárea considerando las malas condiciones obstétricas. Se obtiene recién nacido vivo, masculino de $2380 \mathrm{~g}$, talla $45 \mathrm{~cm}$, Apgar 8-8, con frecuencia cardiaca de 50 a 55 lat $/ \mathrm{min}$, sin acidosis metabólica. EI ECG demostró bloqueo AV completo con disociación auriculo-ventricular, frecuencia cardiaca auricular de 140 a 150 lat/min, y ventricular de 50 a $55 \mathrm{lat} / \mathrm{min}$. Al día siguiente de haber nacido se instala marcapasos unicameral con cable epicárdico ventricular. Es dado de alta al séptimo día de vida en excelentes condiciones generales. Sus controles ambulatorios han demostrado función cardiovascular normal y funcionamiento adecuado del marcapasos. 


\section{DISCUSIÓN}

Ritmo cardiaco. El corazón fetal presenta casi todas las características del corazón adulto ya desde las 8 semanas de gestación. El sistema exito conductor se desarrolla entre las 8 y las 14 semanas. La frecuencia cardiaca fetal es variable según la edad gestacional siendo promedio de $155 \pm 20$ lat $/ \mathrm{min}$ a las 20 semanas, y de $140 \pm 20 \mathrm{lat} / \mathrm{min}$ cerca del término (4).

La regulación de la frecuencia del corazón fetal es similar al corazón del adulto. Tiene un marcapasos, el nódulo sinusal, ubicado en la porción posterior de la desembocadura de la vena cava superior a la aurícula derecha. Existe otro marcapasos con frecuencia mas baja, el nodo aurículo-ventricular, ubicado en la región posterobasal del septum interauricular, cerca del orificio del seno coronario (4).

Gran parte de las arritmias fetales son extrasístoles supraventriculares aisladas que ceden espontáneamente; por el contrario algunas alteraciones del ritmo pueden ser graves y causar hidrops fetal no inmune y muerte fetal. Las arritmias cardiacas se pueden clasificar de acuerdo a la frecuencia cardiaca basal en taquiarritmias o bradiarritmias. Dentro de las primeras se encuentran la taquicardia supraventricular, el flutteratrial, la fibrilación atrial, la taquicardia ventricular y la taquicardia sinusal (4).

Dentro de las bradiarritmias se consideran la bradicardia sinusal y los bloqueos cardiacos que pueden ser de primer grado, segundo grado, o completo. Un bloqueo de primer grado se presenta como una prolongación del intervalo PR, rara vez diagnosticada en el feto. El bloqueo de segundo grado puede ser con prolongación progresiva del intervalo PR, (fenómeno de Wenckebach) o fijo tipo $2 \times 1$. El bloqueo completo es una disociación completa de latido atrial y ventricular, donde el ventrículo late a un ritmo de 40 a 80 lat $/ \mathrm{min}$ independiente de la aurícula (4-6). Al existir un bloqueo AV completo, la aurícula se contrae al ritmo sinusal, pero el impulso no será transmitido al ventrículo, de modo que el ritmo de sístole ventricular queda bajo el dominio del nódulo $\mathrm{AV}$, el que mantiene una frecuencia entre 40 y $80 \mathrm{lat} / \mathrm{min}$ (6).

Es importante diferenciar los casos de bloqueo AV completo según presencia o ausencia de malformaciones cardiacas. Aquellos con presencia de malformación cardiaca representan el $40-60 \%$ de los casos $(1,4)$; el resto, con anatomía cardiaca normal, son casi todos de etiología inmunológica. Hacer esta diferenciación es importante desde el punto de vista pronóstico ya que los bloqueos AV asociados a malformación tienen una sobrevida de solo $5-14 \%$, mientras que aquellos sin malformacio- nes presentan una sobrevida de $89 \%(1,6,7)$.

Etiología inmunológica. El bloqueo completo cardiaco congénito sin malformación asociada tiene como principal factor etiológico la presencia de inmunocomplejos contra ribonucleoproteínas llamadas La y Ro. El sistema de HLA-B8-DR3 y el endovirus humano 3 (ERV-3) son marcadores genéticos que predisponen a la aparición de otros inmunocomplejos que también podrían ser causantes de la enfermedad (8-13).

Los anticuerpos anti-Ro/SS-A y anti-La/SS-B pertenecen a los anticuerpos órganos-no-específico presentes en enfermedades reumatológicas como el Lupus Eritematoso Sistémico (LES) o el Síndrome de Sjögren, junto al factor reumatoideo y otros anticuerpos antinucleares. En células humanas la proteína Ro es una partícula polipeptídica de 60 y $52 \mathrm{kDa}$ (existen al menos dos tipos de Ro) en asociación con una porción de RNA conocida como hY1-5 con 100 nucleótidos de longitud. Por su parte La/SS-B es una proteína de $48 \mathrm{Kd}$ también asociada con el RNA, en zonas llamadas 7S y 5S, y no comparte determinantes antigénicos ni homología con el polipéptido Ro $(3,14,15)$.

Se considera que la presencia de estos anticuerpos (Anti-Ro y Anti-La) es casi un prerrequisito para el bloqueo cardiaco fetal (8). Entre un 60 a $83 \%$ de las madres de los niños afectados tiene anticuerpos anti-Ro circulantes; nótese que solo un $47 \%$ presenta una enfermedad del tejido conectivo activa $(3,8,16)$. Entre los fetos afectados un $80-94 \%$ son positivos para anti-Ro o anti-LA $(10,16)$.

El ERV-3 es una proteína que se expresa en la placenta y en otros tejidos fetales, pero mayormente en el corazón e hígado fetal (4). Se estudió la relación de los anticuerpos anti- La y anti-Ro con el antígeno ERV-3 en dos embarazos de una mujer cuyo primer feto fue afectado por un bloqueo y el segundo fue sano, observándose que el feto comprometido tenia IgGanti-La y ERV-3 positivos, a diferencia del feto sano que presentaba valores menores de anti-La y proteína ERV-3 $(12,17)$.

La proteína ERV-3 esta relacionada con enfermedades autoinmunes con manifestaciones inflamatorias en diferentes órganos. En la mujer embarazada se logra aislar ERV-3 desde las 8 semanas, con valores máximos entre las 11 y 14 semanas. Es abundante en el sinciciotrofoblasto y no se conoce la razón por la cual no produce una reacción inflamatoria en la placenta (18). Un valor mayor a dos desviaciones estándar del promedio se considera anormal. La madre de un feto con bloqueo AV será positiva en el $67 \%$ de las veces para este antígeno (10). 
El gen HLA-B8-DR3 esta relacionado con enfermedades autoinmunes y se lo asocia con la formación de auto anticuerpos. La asociación de HLAB8-DR3 con bloqueo AV fetal fue demostrada en un estudio con 15 madres sin diagnóstico de LES cuyos hijos presentaban bloqueo cardiaco completo (19).

Fisiopatología. Los anticuerpos (IgG) pasan la barrera placentaria mediante fijación a los receptores Fc, siendo transportados por vacuolas a través de la célula trofoblástica y liberados por exocitosis a la sangre fetal (20). Se ha observado in vitro que las IgG 1 y 3 pasan con mayor facilidad la barrera placentaria y se adhieren a los miocitos y sistema de conducción cardiaca $(3,20,21)$. No se ha esclarecido completamente cual de los anticuerpos (anti-Ro $56 \mathrm{Kd}$ o anti-La de $48 \mathrm{Kd}$ ) es el principal responsable de la enfermedad (20).

Una vez en el feto, estos anticuerpos se adhieren a los tejidos fetales siendo las células musculares lisas y el sistema de conducción cardiaco su principal blanco (21). Se postuló que el efecto podría iniciarse en el segundo y tercer trimestre de la vida intrauterina, sin embargo evidencia reciente muestra que el daño se iniciaría en etapa embrionaria $(3,11)$. Un estudio histológico de corazones fetales entre 9 a 25 semanas, todos provenientes de madres con mesenquimopatías y anti Ro positivo tanto en la madre como en el feto, evidenció la presencia de inmunocomplejos en zonas intracelulares del sistema de conducción, a esa edad gestacional. El ingreso de estos grandes anticuerpos (150.000 daltons) al interior de la célula solo se podría explicar por la adherencia a receptores superficiales presentes en dichas células en el período de la organogénesis.

Una vez en la célula cardiaca, los anticuerpos producen una reacción inflamatoria con degeneración fibrosa del sistema de conducción y músculo cardiaco (5). Se ha descrito fibrosis y atrofia del nodo sinusal, fibrosis del septumatrial con evidencia de inflamación crónica (presencia de macrófagos y monocitos), degeneración fibrosa del nodo AV e interrupción del haz de His que está reemplazado por una gran fibrosis (22). Se postuló que existirían dos razones principales para el bloqueo, una la interrupción o división del haz de His, y secundariamente la fibrosis del nodo $\mathrm{AV}$, entidades que deberían ser tomadas por separado. Con respecto al miocardio, la presencia de una miocarditis generalizada llevaría a fibroelastosis con subsecuente insuficiencia cardiaca (22).

Se ha planteado que el bloqueo AV es una enfermedad progresiva, donde la injuria inicial produciría un bloqueo de primer grado, con un alargamiento progresivo del intervalo PR hasta evolucionar a un bloqueo completo AV. Sin embargo, en el estudio PRIDE (23), nos mostró en forma prospectiva la evolución de los fetos de pacientes con anticuerpos SSA-Ro positivo y/o anti SSB-La antes de las 16 semanas. Este estudio descriptivo, mostró que la evaluación semanal de estos fetos no evidenció la evolución del bloqueo AV de primer, segundo y tercer grado, sino que las pacientes afectadas debutaron con bloqueo completo AV.

Diagnóstico. Los bloqueos cardiacos fetales son descubiertos en los controles prenatales, un $82 \%$ antes de las 30 semanas, y en promedio a las 23 semanas (6). La auscultación de los latidos era la forma más común de diagnóstico pero esto ha cambiado desde la década de los 80 dada la disponibilidad de la ecografía rutinaria (6). La bradicardia probablemente se inicia desde las 16 semanas, momento en el cual el sistema de conducción ya esta formado.

Hecho el diagnóstico el enfrentamiento se basa en anamnesis, examen físico, exámenes de laboratorio y métodos auxiliares de diagnóstico como la ecotomografía de tiempo real, modo M y Doppler color pulsado. La anamnesis tiene el objetivo de buscar dirigidamente síntomas sugerentes de enfermedades auto inmunes como el LES y Sjögren; aunque debemos recordar que sólo la mitad de las pacientes tendrán antecedentes de enfermedad del tejido conectivo.

Es también muy importante los antecedentes obstétricos en busca de malformaciones, o bloqueos cardiacos ya que ambas situaciones son recurrentes. Se debe buscar la presencia de anticuerpos anti-Ro, anti-La, dada su asociación y potencial rol causal de la enfermedad. La determinación de los anticuerpos se realiza por medio de técnica de ELISA, inmunofluorescencia indirecta, doble inmunodifusión y látex $(3,9,10)$. Se ha descrito que están presentes en $30-40 \%$ de las pacientes con LES y $70-80 \%$ de las pacientes con síndrome de Sjögren; mientras que la incidencia en pacientes sanas es de $0,44 \%$ (3).

El ecocardiograma bidimensional permite descartar malformaciones anatómicas y evaluar la función sistólica ventricular. El modo $\mathrm{M}$ y el Doppler pulsado son parte importante de la evaluación funcional del corazón. Para considerar un bloqueo como completo se debe observar una disociación AV con un latido atrial a una frecuencia normal o alta y un latido ventricular regular con frecuencias bajas. Esto se logra colocando el modo $M$ en un eje longitudinal del corazón pasando por aurícula, ventrículo y válvulas (6).

El Doppler pulsado nos muestra la válvula mitral con flujo rápido que es reflejo de la contracción 
atrial y a nivel de la salida de la aorta se observa un flujo lento, reflejo de la bradicardia ventricular. La aurícula tiene una contracción regular; si es muy rápida o irregular se debe sospechar de una taquiarritmia auricular asociada a un bloqueo completo. La frecuencia atrial se ha descrito que puede estar entre 96 a 400 lat $/ \mathrm{min}$ y la ventricular entre 40 a 80 $\mathrm{lat} / \mathrm{min}(6)$.

En el estudio PRIDE (23), a una cohorte de pacientes con anticuerpos SSA-Ro y/o anti SSB-La positivo se les hizo evaluación semanal con ecocardiograma y Doppler pulsado. La incidencia de bloqueo fue del $3 \%$ (6 fetos), 3 fetos con bloqueo de primer grado y 3 fetos con bloqueo $\mathrm{AV}$ completo. No se observó evolución en los grados de bloqueo AV, ni se observó diferencia entre la duración del intervalo PR entre los fetos sanos y el grupo con bloqueo AV de tercer grado. Quizás la evaluación semanal no es un tiempo suficiente para detectar bloqueos de conducción AV en estadios más precoces, o bien en los fetos que van a hacer bloqueo completo, no existe esa evolución. Un dato interesante que mostró este estudio es que el promedio de títulos de anticuerpos fue significativamente mayor en las pacientes con bloqueo AV completo.

Tratamiento. El tratamiento prenatal del bloqueo completo AV es un tema controvertido. Dado la baja incidencia de esta enfermedad es difícil hacer trabajos de buen diseño para probar las distintas terapias propuestas. La mayoría de la evidencia disponible son series de casos retrospectivas. Dentro de los tratamientos propuestos están el uso de corticoides, fluorados y no fluorados, betamiméticos, inmunoglobulina e inmunoabsorción extracorpórea. Algunos autores preconizan controles estrictos y frecuentes de las pacientes con anticuerpos anti Ro/SSA y/o anti La/SSB positivos y aquellas con antecedentes de un feto afectado, para detectar bloqueos de bajo grado, para así ofrecer un tratamiento que detenga la progresión.

Parece ser que una vez encontrado el bloqueo completo es imposible revertirlo, posiblemente porque el daño anatómico ya está presente (24). Los corticoides han sido utilizados por sus efectos antiflamatorio e inmunomodulador. Las drogas más utilizadas son la betametasona y dexametasona, ambas capaces de pasar la barrera placentaria. Las dosis sugeridas son de 4-9 mg/día de dexametasona por 3 a 19 semanas, betametasona 12-24 $\mathrm{mg} / \mathrm{semana}$ por mínimo de 6 semanas, o prednisona 10-40 mg/día $(14,15,24,25)$.

Una revisión retrospectiva donde se compara fluoro corticoide (dexametasona y betametasona) versus corticoides no fluorados con un total de 28 y 22 pacientes por grupo, observó que hubo solo una reversión de algunos bloqueos de segundo grado a bloqueo de primer grado, disminución de la regurgitación mitral, resolución de derrame pleural y pericárdico, resolución de ascitis y oligoamnios exclusivamente en el grupo tratado con fluorocortidoides, por lo que en caso de decidir su uso, se debe preferir estas drogas. Destaca que no hubo reversión de los bloqueos AV completos (25).

Algunos trabajos apoyan la idea de tratar lo más precozmente posible, al detectar bloqueo de primer grado. En el estudio PRIDE (23), se trataron con dexametasona a los seis fetos de la cohorte que desarrollaron bloqueo. Dos fetos con bloqueo completo murieron in utero con hidrops fetal y el otro necesitó la instalación de marcapasos al nacer. De los que desarrollaron bloqueo de primer grado, dos revirtieron a ritmo sinusal y el tercero revirtió al año de vida. Dado la baja incidencia de la enfermedad y el diseño del estudio, no se puede concluir si los pacientes con bloqueo de primer grado revirtieron por el efecto de la droga.

En otro estudio prospectivo (26), se siguieron 70 fetos con riesgo de desarrollar bloqueo AV. Seis fetos fueron afectados con bloqueo AV de primer grado y fueron tratados con dexamentasona, los 6 revirtieron a ritmo sinusal dentro de los 4 a 14 días después de iniciado el tratamiento. En esta serie no hubo fetos con bloqueo completo AV. El diseño de este estudio no consideraba probar distintas terapias, por lo que no se pueden sacar conclusiones sobre la efectividad de la dexametasona en el tratamiento de estos fetos.

Otra corriente de pensamiento señala que una vez realizado el diagnóstico el manejo depende de los hallazgos ecográficos; si el único hallazgo es una bradicardia fetal, descartándose malformaciones asociadas, se procede a evaluaciones seriadas $\sin$ ninguna intervención hasta el término del embarazo, a menos que se evidencie signos sugerentes de insuficiencia cardiaca. Es aconsejable derivar a un hospital de nivel terciario para su interrupción programada y una adecuada atención neonatal y por cardiólogo pediatra. El parto vaginal no esta contraindicado y se debe proceder a operación cesárea solo en caso de indicación obstétrica. Durante el parto es importante la monitorización continua ya que un cambio en la línea basal ó presencia de meconio indican un probable sufrimiento fetal aconsejándose la interrupción por vía alta. La utilización de oximetría de pulso y $\mathrm{pH}$ de cuero cabelludo son claves en determinar la condición fetal durante el trabajo de parto y su uso debe ser recomendado $(27,28)$.

Otro escenario es el de fetos con compromiso hemodinámico y con presencia de bradicardias extremas en donde es necesario intentar algún 
procedimiento para mejorar la condición fetal. Las indicaciones para el uso de corticoides en estos embarazos serían las siguientes: hidrops fetal, hallazgo reciente del bloqueo, derrames en serosas, arritmias fetales inestables $(14,15,25)$. Con el mismo efecto inmunomodulador se ha utilizado inmunoglobulina; el uso combinado profiláctico de corticoides e inmunoglobulina logró disminuir el número de anticuerpos y el riesgo de bloqueo $\mathrm{AV}$ en una madre con antecedentes de hijos previos con esta patología (29).

Los betamiméticos son otro grupo de drogas utilizadas con el objeto de mejorar la bradicardia. Entre ellos encontramos la isoprenalina, salbutamol, terbutalina, isoproterenol y ritodrina; la evidencia muestra que los betamiméticos logran aumentar el ritmo ventricular en 15 a 50\% (6). Sin embargo, el aumento de la frecuencia cardiaca fetal no traduce un claro beneficio para el feto, por lo que su uso no es recomendado. Existen también tratamientos invasivos consistentes en el uso de inmunoglobulinas y plasmaféresis de la madre y la colocación de un marcapaso fetal in útero. Los resultados del uso de marcapaso son exitosos, pero a su vez de mucha complejidad y frecuentes complicaciones como rotura de membranas y muerte fetal, por lo que deben considerarse como un último recurso cuando los otros tratamientos han fracasado y se considera que el feto está en alto riesgo de muerte $(3,6,25)$.

Después del nacimiento debe evaluarse la necesidad de insertar un marcapasos. Este procedimiento será necesario en por lo menos un $63 \%$ de los recién nacidos, de los cuales $52 \%$ lo requieren dentro de la primera semana, $23 \%$ antes del año y $25 \%$ después del primer año de vida $(6,17)$. Hoy en día la instalación de un marcapaso es un procedimiento relativamente simple y que claramente mejora la función ventricular, por lo que la mayoría de los recién nacidos deberían recibirlo antes del alta.

Pronóstico. Un 8 a $20 \%$ de los niños con bloqueo AV mueren, la mayoría antes de los 28 días. Entre los signos de mal pronóstico se cuentan latidos atriales menores a 120 por minuto y ventriculares menores a 55 por minuto, la frecuencia promedio entre ambos menor de 127 latidos por minuto, presencia de hidrops fetal y la falta de respuesta a los corticoides $(2,6,8)$. La causa principal de muerte es la falla cardiaca secundaria a una miocardiopatía degenerativa $(13,17)$.

No se conoce por qué solo el $5 \%$ de las pacientes anti Ro positivo evolucionan con bloqueo cardiaco fetal, ni por qué de ellas solo el $16 \%$ recurren (9). De todos estos, los más relacionados con bloqueo cardiaco fetal son en orden de importancia el anti-Ro $56 \mathrm{Kd}(80 \%)$, el anti-La $48 \mathrm{Kd}(80 \%)$ y el
anti-Ro $60 \mathrm{Kd}$ (55\%) otorgando la presencia de los dos primeros un riesgo 35 veces mayor que la población general de desarrollar la enfermedad (20). En un estudio retrospectivo de 33 madres con antecedentes de bloqueo cardiaco en embarazos anteriores, se concluye que la presencia de anti Ro de $56 \mathrm{Kd}$ entre otros anticuerpos confiere el mayor riesgo de desarrollar la enfermedad (14).

\section{CONCLUSIÓN}

El bloqueo fetal cardiaco es un tipo de arritmia poco frecuente. Tiene estrecha relación con mesenquimopatía materna y la presencia de anticuerpos anti-Ro y anti-La. Actualmente el diagnóstico se hace con frecuencia en el segundo trimestre y durante un examen ecográfico de rutina. En ese momento se debe realizar un estudio de anatomía cardiaca fetal para poder distinguirla de los bloqueos secundarios a malformaciones. El ecocardiograma bidimensional con Doppler fetal permite además evaluar el tipo de bloqueo y sus consecuencias en la función cardiaca fetal, la que de estar muy alterada puede llevar a un hidrops fetal. El manejo es expectante en la mayoría de los casos ya que no existe forma de revertir el bloqueo. A pesar que existen reportes que apoyarían el tratamiento precoz de esta patología, no existe evidencia concluyente para apoyar el tratamiento con corticoides a todos estos fetos. En caso de presentar una descompensación hemodinámica, es posible administrar corticoides como primera medida terapéutica con un éxito limitado. Existen otras opciones terapéuticas con resultados inciertos; hasta el momento no se han realizado estudios prospectivos controlados que permitan demostrar la efectividad del tratamiento médico en esta patología fetal. No existe contraindicación del parto vaginal y el uso de $\mathrm{pH}$ de cuero cabelludo y oximetría de pulso parecen ser métodos adecuados para la evaluación de la condición fetal intraparto. Es importante remarcar que estos embarazos deben ser controlados por un equipo multidisciplinario, constituidos por perinatólogo, ecografista, neonatólogo y cardiólogo pediatra; este enfoque permitiría evitar intervenciones innecesarias, anticipar los riesgos fetales y obtener un mejor pronóstico postnatal. Dada la ausencia de información definitiva, recomendamos que cada unidad determine un protocolo específico de tratamiento de los casos de bloqueo AV y de seguimiento de pacientes en riesgo. Es altamente relevante diferenciar entre la paciente sin historia previa, portadora de anticuerpos anti-Ro y anti-La positivos (bajo riesgo de bloqueo, $<5 \%$ ), de aquella paciente con anticuerpos positivos e historia de un 
hijo previo afectado (alto riesgo de recurrencia, cercano al $20 \%$ ). En nuestra unidad recomendamos el seguimiento con medición semanal del intervalo PR en el grupo de pacientes con historia previa, iniciando corticoides en caso de detectar una prolongación del intervalo PR. En pacientes sin historia previa, recomendamos solo control clínico.

\section{BIBLIOGRAFÍA}

1. McCurdy CJ, Reed K. Fetal Arrhythmias. En: Copel JA, Reed KL, eds. Doppler Ultrasound in Obstetrics and Gynecology. New York: Raven Press Ltd., 1995; 253-69.

2. Michaelsson M, Engle MA. Congenital complete heart block: an international study of the natural history. Cardiovasc Clin 1972;4:85-101.

3. Gutierrez M, Figueroa F, Rivero S, et al. [Maternal connective tissue disease associated with congenital AV block]. Rev Med Chil 1989;117:789-93.

4. Parer J. Heart Rate. En: Creasy R, Resnik R, eds. Maternal-Fetal Medicine. Philadelphia: W.B. Saunders Company, 1999; 270-99.

5. Schmidt KG, Ulmer HE, Silverman NH, Kleinman CS, Copel JA. Perinatal outcome of fetal complete atrioventricular block: a multicenter experience. J Am Coll Cardiol 1991;17:1360-6.

6. Silverman N, Schmidt RG. [Ecographic Evaluation of the Fetal Heart].En: Callen P, ed. [Ecography in Obstetrics and Gynecology].Madrid: Panamericana S.A., 1995; 314-58.

7. Buyon JP, Hiebert R, Copel J, et al. Autoimmune-associated congenital heart block: demographics, mortality, morbidity and recurrence rates obtained from a national neonatal lupus registry. J Am Coll Cardiol 1998;31:1658-66.

8. Figueroa J, Tapia P, Ruiz M, Ocaranza M, Henriquez G. [Prenatal diagnosis and management of one case of complete atrio-ventricular block]. Rev Chil Obstet Ginecol 1997;62:453-7.

9. Julkunen $\mathrm{H}$, Kurki $\mathrm{P}$, Kaaja $\mathrm{R}$, et al. Isolated congenital heart block. Long-term outcome of mothers and characterization of the immune response to SS-A/Ro and to SS-B/La. Arthritis Rheum 1993;36:1588-98.

10. Gordon PA, Khamashta MA, Hughes GR, Rosenthal $E$. Increase in the heart rate-corrected QT interval in children of anti-Ro-positive mothers, with a further increase in those with siblings with congenital heart block: comment on the article by Cimaz et al. Arthritis Rheum 2001;44:242-3.

11. Groves AM, Allan LD, Rosenthal E. Outcome of isolated congenital complete heart block diagnosed in utero. Heart 1996;75:190-4.

12. Horsfall AC, Neu E, Forrest G, Venables PJ, Field M. Maternal autoantibodies and congenital heart block: clues from two consecutive pregnancies, one in which there was congenital complete heart block and one in which the fetus was healthy. Arthritis Rheum
1998;41:2079-80.

13. Rosenthal D, Druzin M, Chin C, Dubin A. A new therapeutic approach to the fetus with congenital complete heart block: preemptive, targeted therapy with dexamethasone. Obstet Gynecol 1998;92:689-91.

14. Silverman E, Mamula M, Hardin JA, Laxer R. Importance of the immune response to the Ro/La particle in the development of congenital heart block and neonatal lupus erythematosus. J Rheumatol 1991;18:120-4.

15. Buyon JP, Swersky SH, Fox HE, Bierman FZ, Winchester RJ. Intrauterine therapy for presumptive fetal myocarditis with acquired heart block due to systemic lupus erythematosus. Experience in a mother with a predominance of SS-B (La) antibodies. Arthritis Rheum 1987;30:44-9.

16. Carter JB, Blieden LC, Edwards JE. Congenital heart block.Anatomic correlations and review of the literature. Arch Pathol 1974;97:51-7.

17. Moak JP, Barron KS, Hougen TJ, et al. Congenital heart block: development of late-onset cardiomyopathy, a previously underappreciated sequela. J Am Coll Cardiol 2001;37:238-42.

18. Venables PJ, Brookes SM, Griffiths D, Weiss RA, Boyd MT. Abundance of an endogenous retroviral envelope protein in placental trophoblasts suggests a biological function. Virology 1995;211:589-92.

19. Vazquez JJ, Garcia J, Gial A, Sobrino JA, Arnalich FJ, Arnaiz A. Complete heart block and the HLA system. Ann Intern Med 1982;96:126.

20. Buyon JP, Winchester R. Congenital complete heart block. A human model of passively acquired autoimmune injury. Arthritis Rheum 1990;33:609-14.

21. Deng JS, Bair LW, Jr., Shen-Schwarz S, RamseyGoldman R, Medsger T, Jr. Localization of Ro (SSA) antigen in the cardiac conduction system. Arthritis Rheum 1987;30:1232-8.

22. Li JM, Fan WS, Horsfall AC, et al. The expression of human endogenous retrovirus-3 in fetal cardiac tissue and antibodies in congenital heart block. Clin Exp Immunol 1996;104:388-93.

23. Friedman DM, Kim MY, Copel JA, et al. Utility of cardiac monitoring in fetuses at risk for congenital heart block: The PR intervaland dexamethasone evaluation (PRIDE) prospective study. Circulation 2008;117;485-93.

24. Copel JA, Buyon JP, Kleinman CS. Successful in utero therapy of fetal heart block. Am J Obstet Gynecol 1995;173:1384-90.

25. Saleeb S, Copel J, Friedman D, Buyon JP. Comparison of treatment with fluorinated glucocorticoids to the natural history of autoantibody-associated congenital heart block: retrospective review of the research registry for neonatal lupus. Arthritis Rheum 1999;42:2335-45.

26. Rein AJ, Mevorach D, Perles Z, Gavri S, Nadjari M, Nir A, Elchalal U. Early diagnosis and treatment of atrioventricular block in thefetus exposed to maternal anti-SSA/Ro-SSB/La antibodies. Circulation. 2009;119:1867-72.

27. Begg L, East C, Chan FY, Brennecke S. Intrapartum fetal oxygen saturation monitoring in congenital fetal heart block. Aust N Z J Obstet Gynaecol 1998;38:271-4. 
28. Van den Berg PP, Nijland R, van den Brand SF, Jongsma HW, Nijhuis JG. Intrapartum fetal surveillance of congenital heart block with pulse oximetry. Obstet Gynecol 1994;84:683-6.
29. Kaaja R, Julkunen H, Ammala P, Teppo AM, Kurki P. Congenital heart block: successful prophylactic treatment with intravenous gamma globulin and corticosteroid therapy. Am J Obstet Gynecol 1991;165:1333-4. 